\title{
Resistance Patterns of Escherichia coli and Klebsiella pneumoniae Bacteria Against Amikacin, Ceftazidime, Meropenem, Nitrofurantoin Antibiotics in Elderly Patients with UTI in Dr. Soetomo General Hospital Surabaya
}

\author{
Herdiyanti ${ }^{1}$, Lindawati Alimsardjono ${ }^{2^{\star}}$, Danti Nur Indiastuti ${ }^{3}$
}

\footnotetext{
${ }^{1}$ Faculty of Medicine, Universitas Airlangga, Surabaya.

2 Department of Medical Microbiology, Faculty of Medicine, Universitas Airlangga, Surabaya.

${ }^{3}$ Department of Pharmacology, Faculty of Medicine, Universitas Airlangga, Surabaya.
}

\begin{abstract}
A B S T R A C T
Introduction: Urinary tract infection (UTI) is an infection that involves the urinary tract, including the urethra, bladder, ureter, and kidneys. UTI is caused by microorganisms that breed in the urinary tract which are supposed to be free of microorganisms. Two bacterias that cause UTI in general are Escherichia coli and Klebsiella pneumoniae. UTI can occur at any age but generally occurs in the elderly. UTI caused by gram-negative bacteria can be killed with several examples of antibiotics namely amikacin, ceftazidime, meropenem and nitrofurantoin. These antibiotics found resistance to the bacteria that cause the UTI. This research is to determine the resistance patterns of Escherichia coli and Klebsiella pneumoniae bacteria against amikacin, ceftazidime, meropenem, nitrofurantoin antibiotics in elderly patients with UTI in Dr. Soetomo Surabaya January-June 2017 period.
\end{abstract}

Methods: A descriptive retrospective study in accordance with the logbook data of Clinical Microbiology Installation at Dr. Soetomo General Hospital Surabaya. Data from this study were taken from all populations that met the inclusion criteria. The results are presented by tables.

Results: There are 163 elderly patient data which diagnosed with urinary tract infection. Escherichia coli resistance pattern against ceftazidime (75.6\%), nitrofurantoin (12.6\%) and meropenem (2.4\%). Meanwhile, Klebsiella pneumoniae against ceftazidime $(72.2 \%)$, nitrofurantoin $(55.6 \%)$, meropenem $(11.1 \%)$ and amikacin (2.8\%).

Conclusion: There were resistance numbers of Escherichia coli and Klebsiella pneumoniae bacteria against antibiotics amikacin, ceftazidime, meropenem, nitrofurantoin in elderly patients with UTI.

\footnotetext{
*Correspondence: lindawatia@gmail.com

JUXTA: Jurnal IImiah Mahasiswa Kedokteran Universitas Airlangga

p-ISSN: 1907-3623; e-ISSN: 2684-9453
}

DOI: $10.20473 /$ juxta.V10I12019.20-24

Open access under Creative Commons Attribution-ShareAlike 4.0 International License (CC-BY-SA) C) (ㄱ)
ARTICLE INFO

Article history:

Received 05 December 2018

Received in revised form 22 January 2019

Accepted 24 January 2019

\section{Keywords:}

Escherichia coli,

Klebsiella pneumonia,

Amikacin,

Nitrofurantoin,

Urinary tract infection. 


\section{Introduction}

Urinary tract infection (UTI) is an infection that involves the urinary tract, including the urethra, bladder, ureter, and kidneys. UTI is caused by microorganisms that multiply in the urinary tract which are supposed to be free of microorganisms ${ }^{1}$. Two bacteria that cause UTI in general are Escherichia coli and Klebsiella pneumoniae ${ }^{2}$. In Ethiopia also found gram-negative bacteria for UTI patients in the first order, namely Escherichia coli (33.3\%) followed by Klebsiella pneumoniae $(19 \%)^{3}$. Whereas in France, E. coli $(73 \%)$ and Klebsiella spp. $(6 \%)^{4}$.

UTI can occur at any age but generally occurs in the elderly. This infection is the second highest infection after respiratory infection in elderly parents ${ }^{5}$. Elderly patients often cannot explain or report complaints that they experience clearly. This causes the infection is often overlooked so that patients experience delays in treatment and can cause complications in the form of sepsis. In addition, all infectious diseases can accelerate the progress of dementia which is a condition in which the ability of a person's brain to decline. Therefore, UTI disease needs to be identified and treated quickly6

UTI caused by gram-negative bacteria can be killed with several examples of antibiotics such as aminoglycosides such as amikacin, cephalosporins such as ceftazidime, carbapenem such as meropenem, and nitrofuran synthesis such as nitrofurantoin. Excessive treatment with antibiotics for UTI is suspected remains a significant problem and causes various negative consequences including the development of microorganisms that are resistant to antibiotic drugs ${ }^{7}$.

Amikacin found resistance to several strains gramnegative bacteria associated with the antibiotic enzyme ${ }^{8}$. Ceftazidime which extends across the blood-brain barrier is also still misused for the treatment of gram-negative bacterial infections whereas this can lead to resistance ${ }^{9}$. Active or sensitive nitrofurantoin to fight Escherichia coli strains was found to be resistant to gram-negative bacteria ${ }^{10}$. The carbapenem antibiotic group including meropenem has begun to be resistant to Enterobacteriaceae ${ }^{11}$. Therefore, the researchers wanted to know the clarity of the resistance patterns of Escherichia coli and Klebsiella pneumoniae bacteria against the antibiotics amikacin, ceftazidime, meropenem, and nitrofurantoin whether they have experienced resistance or are still sensitive to their use in elderly patients with UTI, especially in Dr. Soetomo January 2017-June 2017 period.

\section{Methods}

This research was a descriptive retrospective. The independent variables were Escherichia coli bacteria, Klebsiella pneumoniae bacteria, age, UTI patient, while the dependent variables were amikacin, ceftazidime, meropenem, and nitrofurantoin.
This research used logbook data with inclusion data (data on male and female patients aged $\geq 60$ years, data on elderly patients suffering from UTI, data on elderly patients suffering from UTI with the causes of the bacteria Escherichia coli and Klebsiella pneumoniae, antibiotic data for amikacin, ceftazidime, meropenem and nitrofurantoin, treated in the period January - June 2017) and exclusion data (patient data that is not complete). The population was patients data of UTI in elderly in Dr. Soetomo General Hospital Surabaya January 2017-June 2017 period. The research sample was taken from the population of patients who were suitable with the inclusion criteria in Dr. Soetomo General Hospital Surabaya in January 2017-June 2017 period which has been done by urine culture test and antibiotic resistance test and recorded in logbook data at the Clinical Microbiology Installation at Dr. Soetomo General Hospital Surabaya. This research took place in Clinical Microbiology Installation at Dr. Soetomo General Hospital Surabaya with January 2017-June 2017 period. The results of this research were presented by tables.

\section{Results}

Table 5.1 Distribution of Research Subject Patterns Based on Gender

\begin{tabular}{lcc}
\hline Gender & $\begin{array}{c}\boldsymbol{n} \\
\text { (Amount) }\end{array}$ & $\begin{array}{c}\text { \% } \\
\text { (Persentage) }\end{array}$ \\
\hline Male & 76 & 46.7 \\
Female & 87 & 53.3 \\
Total & 163 & 100.0 \\
\hline
\end{tabular}

In table 5.1 shows that from 163 patient data obtained a few more subjects of women who suffer from UTI as many as 87 patient data $(53.5 \%)$ compared to male subjects which amounted to 76 patient data (46.7\%).

Table 5.2 Distribution of Age Subject Research Patterns

\begin{tabular}{ccc}
\hline Age & $\begin{array}{c}\boldsymbol{n} \\
\text { (Amount) }\end{array}$ & $\begin{array}{c}\% \\
\text { (Persentage) }\end{array}$ \\
\hline $60-70$ & 109 & 66.9 \\
$71-80$ & 44 & 27 \\
$81-90$ & 10 & 6.1 \\
Total & 163 & 100.0 \\
\hline
\end{tabular}

In table 5.2 shows that the data of UTI patients in elderly aged $\geq 60$ years is 163 patient data. The most data of UTI patients were in the 60-70 year age group, which were 109 patient data $(66.9 \%)$.

Table 5.3 Distribution of Research Subject Patterns Based on Bacteria Causes of UTI 
In table 5.3 shows that the bacteria that cause UTI in the elderly were found to be the most, namely Escherichia coli bacteria with 127 isolates $(78 \%)$ followed by Klebsiella pneumoniae with 36 isolates (22\%).

Table 5.4 Distribution of Research Subject Patterns Based on Escherichia coli Bacteria on Amikacin, Ceftazidime, Meropenem and Nitrofurantoin Antibiotics in UTI Patients

\begin{tabular}{lcccccc}
\hline Antibiotic & \multicolumn{6}{c}{ Escherichia coli $(\boldsymbol{n}=\mathbf{1 2 7})$} \\
\cline { 2 - 7 } & $\mathbf{R}$ & $\%$ & $\mathbf{I}$ & $\%$ & $\mathbf{S}$ & $\%$ \\
\hline Amikacin & 0 & 0 & 1 & 0.8 & 126 & 99.2 \\
Ceftazidime & 96 & 75.6 & 0 & 0 & 31 & 24.4 \\
Meropenem & 3 & 2.4 & 0 & 0 & 124 & 97.7 \\
Nitrofurantoin & 16 & 12.6 & 9 & 7.1 & 102 & 80.3 \\
\hline
\end{tabular}

In Table 5.4 shows that the resistance pattern of Escherichia coli bacteria to ceftazidime antibiotics has the highest resistance level $(75.6 \%)$. The next highest followed by nitrofurantoin antibiotics (12.6\%), meropenem (2.4\%). Whereas for antibiotics amikacin does not have a resistance number $(0 \%)$.

\begin{tabular}{lllllll}
\hline \multirow{2}{*}{ Antibiotic } & \multicolumn{6}{l}{ Klebsiella pneumoniae $(n=36)$} \\
\cline { 2 - 7 } & $\mathrm{R}$ & $\%$ & $\mathrm{I}$ & $\%$ & $\mathrm{~S}$ & $\%$ \\
\hline Amikacin & 1 & 2.8 & 0 & 0 & 35 & 97.2 \\
Ceftazidime & 26 & 72.2 & 0 & 0 & 10 & 27.8 \\
Meropenem & 4 & 11.1 & 0 & 0 & 32 & 88.9 \\
Nitrofurantoin & 20 & 55.6 & 6 & 16.7 & 10 & 27.8 \\
\hline
\end{tabular}

Table 5.5 Distribution of Research Subject Patterns Based on Klebsiella pneumoniae Bacteria on Amikacin, Ceftazidime, Meropenem and Nitrofurantoin Antibiotics in UTI Patients

In Table 5.5 shows the resistance pattern of Klebsiella pneumoniae bacteria to ceftazidime antibiotics has the highest resistance rate $(72.2 \%)$. Then the next highest followed by nitrofurantoin antibiotics (55.6\%), meropenem $(11.1 \%)$ and amikacin $(2.8 \%)$.

\section{Discussion}

The results showed that the data of patients with subjects of women who suffered from UTI were slightly more than male subjects. The dominant UTI in women can be caused by differences in the anatomical structure of a woman's urethra shorter than a man's, making it easier for bacteria to enter the woman's bladder. Female bladder is also closer to the anus which makes susceptible bacteria move from the anus to the urethra ${ }^{12}$.

The results of the study explained that every elderly population starting from 60 years to 90 years had data that they included UTI patients. UTI is one of the most common infections in geriatric or elderly patients. UTI can occur at certain times that occur in the elderly. This infection is the second highest infection after respiratory infection in the elderly ${ }^{13}$.

\begin{tabular}{lcc}
\hline \multicolumn{1}{c}{ Bacteria } & $\begin{array}{c}\boldsymbol{n} \\
\text { (Amount) }\end{array}$ & $\begin{array}{c}\text { \% } \\
\text { (Persentage) }\end{array}$ \\
\hline Escherichia coli & 127 & 78.0 \\
Klebsiella pneumoniae & 36 & 22.0 \\
Total & 163 & 100.0
\end{tabular}

The most bacteria found in elderly patients with UTI are Escherichia coli bacteria followed by Klebsiella pneumoniae. The most common bacteria that causes UTI is Escherichia coli then the second most bacterial, Klebsiella pneumoniae ${ }^{14}$. This is due to the large intestine and perineal area functioning as a reservoir for pathogenic bacteria such as Escherichia colit. In addition, most of the UTIs involving the bladder or kidneys in a healthy host are caused by the small number of type $\mathrm{O}$ antigens that can produce colonization and cause infection. Escherichia coli bacteria are used for uropathogenic and can produce cytotoxic hemolysin then cause tissue invasion. ${ }^{15}$

The pattern of resistance of Escherichia coli bacteria obtained from the results of this study shows the highest resistance results, namely Escherichia coli bacteria against ceftazidime antibiotics. The next highest followed by the antibiotic nitrofurantoin and antibiotics meropenem. Whereas antibiotics amikacin does not have resistance numbers. The resistance of Escherichia coli bacteria to ceftazidime antibiotics can be caused by Escherichia coli bacteria which have been found to have a resistance mechanism in the ExtendedSpectrum Beta-Lactamase (ESBL) with porin impermeability and efflux pump activity ${ }^{16}$. Antibiotic resistance of nitrofurantoin to Escherichia coli bacteria is associated primarily with the presence of mutations in the chromosomal nitroreductase gene (nfsA and nfsB) involved in converting drugs into toxic compounds. In addition, nitrofurantoin resistance can also be caused by the presence of the oqxAB gene mediated by plasmids ${ }^{17}$.

The resistance mechanism of carbapenem antibiotics is caused by carbapenemase production and also due to deletion or loss of outer protein membranes $^{18}$. Amikacin can actively fight against Escherichia coli by transporting through the cytoplasmic membrane which in the process depends on the available oxygen. This antibiotic also has a bactericidal effect on gram-negative bacteria including Escherichia coli so that amikacin in its use is sensitive to Escherichia coli. ${ }^{19}$ This study explains that the resistance pattern of Escherichia coli bacteria to ceftazidime, nitrofurantoin and meropenem in elderly patients with UTI can be known to cause the resistance in general, which is caused by mutations in genes, changes in permeability (membranes and porins), production of enzymes that can damage antibiotics, changes of efflux pump activity and the use of antibiotic abuse.

The resistance pattern of Klebsiella pneumoniae bacteria obtained from the results of this study shows that the highest level of resistance of Klebsiella pneumoniae is against ceftazidime antibiotics. 
The next highest sequence is followed by nitrofurantoin, meropenem, and amikacin antibiotics.

The resistance of ceftazidime against Klebsiella pneumoniae can be caused by the extended-spectrum betalactamase gene (ESBL) which is a beta-lactamase that has mutations and can damage ceftazidime antibiotics by ceftazidime binding to bacterial enzymes ( $\beta$-lactamase) which can hydrolyze the ring $\beta$ - lactam and deactivate the antibacterial properties of ceftazidime antibiotics.

Nitrofurantoin resistance is caused mainly by the presence of mutations in nfsA or nfsB which both will affect nitroreductase which is not sensitive to oxygen so that nitrofurantoin resistance can increase. In addition, the presence of the oqxAB gene in Klebsiella pneumoniae can increase its resistance to the antibiotic nitrofurantoin ${ }^{20}$. The resistance of meropenem against Klebsiella pneumoniae caused by the NDM-1 gene which is a carbapenemase gene encoding ${ }^{21}$. In addition, the OXA-181 enzyme can affect carbapenemase so meropenem resistance against Klebsiella pneumoniae can also occur ${ }^{22}$.

Mechanism resistance of amikacin against Klebsiella pneumoniae caused by the enzymatic modification of the drug, modification of ribosomal target and decreased intracellular antibiotic accumulation by changing the permeability of the outer membrane, decreased transport of the inner membrane or active efflux ${ }^{23}$. Among all the mechanism resistance, the most common amikacin mechanism resistance caused by the production of aminoglycoside-modifying enzymes ${ }^{23}$. This study explains that the resistance pattern of Klebsiella pneumoniae bacteria against ceftazidime, nitrofurantoin, meropenem and amikacin in elderly UTI patients in general can be caused by the production of enzymes that can damage antibiotics, inhibited peptidoglycan synthesis, modification of ribosomal targets, changes in permeability membranes, decreased membrane transport and mutations in genes.

\section{Conclusion}

Based on this research, the number of data for elderly patients suffering from UTI is 163 patient data. The number of data for elderly patients of women with UTI was a little more than 87 patient data $(53.3 \%)$ compared to the data of 76 elderly male patients (46.7\%). The bacteria that cause UTI in the most elderly are Escherichia coli bacteria with a total of 127 isolates (78\%) followed by Klebsiella pneumoniae with 36 isolates (22\%). Escherichia coli bacteria are resistant to antibiotics with the highest resistance number of ceftazidime $(75.6 \%)$, nitrofurantoin $(12.6 \%)$ and meropenem $(2.4 \%)$. Whereas amikacin antibiotics do not have resistance numbers $(0 \%)$.

Klebsiella pneumoniae bacteria are resistant to antibiotics with the highest resistance number of ceftazidime $(72.2 \%)$, nitrofurantoin $(55.6 \%)$, meropenem $(11.1 \%)$ and amikacin (2.8\%).

\section{CONFLICT OF INTEREST}

The author stated there is no conflict of interest

\section{REFERENCES}

1. Urinary Tract Infection (UTI). 2017

2. Flores-Mireles $\mathrm{AL}$, Walker $\mathrm{JN}$, Caparon $\mathrm{M}$ and Hultgren SJ. Urinary Tract Infections: Epidemiology, Mechanisms of Infection and Treatment Options. Nature Reviews Microbiology. 2015; 13: 269-84.

3. Beyene $G$ and Tsegaye W. Bacterial Uropathogens in Urinary Tract Infection and Antibiotic Susceptibility Pattern in Jimma University Specialized Hospital, Southwest Ethiopia. Ethiopian Journal of Health Sciences. 2011; 21: 141-6.

4. Malmartel A and Ghasarossian C. Epidemiology of Urinary Tract Infections, Bacterial Species and Resistances in Primary Care in France. European Journal of Clinical Microbiology \& Infectious Diseases : Official Publication of the European Society of Clinical Microbiology. 2016; 35: 447-51.

5. Rowe TA and Juthani-Mehta M. Urinary Tract Infection in Older Adults. Aging Health. 2013; 9.

6. Urinary Tract Infection. National Association For Continence. 2017.

7. Hisano $M$, Bruschini $H$, Carlos Nicodemo $A$ and Srougi M. Uncomplicated Urinary Tract Infections in Women in a Sao Paulo Quaternary Care Hospital: Bacterial Spectrum and Susceptibility Patterns. 2014, p.98-108.

8. Hisano M, Bruschini $H$, Nicodemo $A C$ and Srougi $M$. Uncomplicated Urinary Tract Infections in Women in a Sao Paulo Quaternary Care Hospital: Bacterial Spectrum and Susceptibility Patterns. Antibiotics (Basel, Switzerland). 2014; 3: 98-108.

9. Ramirez SM and Tolmasky EM. Amikacin: Uses, Resistance, and Prospects for Inhibition. Molecules. $2017 ; 22$

10. Trevor A KB, Kruediring M. Katzung \& Trevor's Pharmacology: Examination \& Board Review. . 11th ed. Philadelpia: PA.: McGraw-Hill, 2015.

11. Laurence L. Brunton RH-D, Björn C. Knollmann. Goodman \& Gilman's: The Pharmacological Basis of Therapeutics, 13e. 13th ed.: Cenveo® Publisher Services., 2018.

12. Mental Health of Older Adults. World Health Organization. 2017.

13. Minardi D, d'Anzeo G, Cantoro D, Conti A and Muzzonigro G. Urinary Tract Infections in Women: Etiology and Treatment Options. International Journal of General Medicine. 2011; 4: 333-43.

14. Gavazzi G, Delerce E, Cambau E, et al. Diagnostic Criteria for Urinary Tract Infection in Hospitalized Elderly Patients Over 75 years of Age: A Multicenter Cross-Sectional Study. Medecine et Maladies Infectieuses. 2013; 43: 189-94.

15. Behzadi P, Behzadi E, Yazdanbod H, Aghapour R, Akbari Cheshmeh M and Salehian Omran D. A Survey on Urinary Tract Infections Associated with the Three Most Common Uropathogenic Bacteria. Maedica. 2010; 5: 111-5.

16. Jawetz, Melnick, \& Adelberg's Medical Microbiology, Twenty-Sixth Edition. 26th ed.: The McGraw-Hill Companies, Inc, 2013.

17. Baroud M, Dandache I, Araj GF, et al. Underlying Mechanisms of Carbapenem Resistance in ExtendedSpectrum Beta-Lactamase-Producing Klebsiella Pneumoniae and Escherichia Coli Isolates at A Tertiary Care Centre in Lebanon: Role of OXA-48 and NDM-1 Carbapenemases. International Journal of Antimicrobial Agents. 2013; 41: 75-9. 
18. Ho PL, Ng KY, Lo WU, et al. Plasmid-Mediated OqxAB Is an Important Mechanism for Nitrofurantoin Resistance in Escherichia coli. Antimicrob Agents Chemother. 2016; 60: 537-43.

19. Ye Y, Xu L, Han Y, Chen Z, Liu C and Ming L. Mechanism for Carbapenem Resistance of Clinical Enterobacteriaceae Isolates. Experimental and Therapeutic Medicine. 2018; 15: 1143-9.

20. Magdalena Fidecka-Skwarzynska MJ, Lucyna Maziarczyk, Anna Malm. A Preliminary Report on the Susceptibility to Aminoglycosides of Escherichia coli Isolated from the Community-Acquired Urinary Tract Infections in Adults in South-east Poland. Current Issues in Pharmacy and Medical Sciences. 2015; 28: 27-9.
21.

Osei Sekyere J. Genomic Insights into Nitrofurantoin Resistance Mechanisms and Epidemiology in Clinical Enterobacteriaceae. Future Science OA. 2018; 4: FSO293-FSO

22. Sharma A, Devi Y, Gopi R, Anandan S, Philip Verghese V and Veeraraghavan B. Mechanisms of Carbapenem Resistance in K.pneumoniae and E. coli from Bloodstream Infections in India. 2016, p.293.

23. Liang $C$, Xing $B$, Yang $X$, Fu $Y$, Feng $Y$ and Zhang $Y$. Molecular Epidemiology of Aminoglycosides Resistance on Klebsiella Pneumonia in a Hospital in China. International journal of clinical and experimental medicine. 2015; 8: 13815. 